\title{
Development of Lung Epithelium from Induced Pluripotent Stem Cells
}

\author{
Mahboobe Ghaedi • Laura E. Niklason • \\ Jordana C. Williams
}

Published online: 30 January 2015

(C) Springer International Publishing AG 2015

\begin{abstract}
Considerable progress has been made in the field of in vitro development of alveolar epithelium from induced pluripotent stem cells. Patient specific derived alveolar cells could potentially populate tissue engineered lungs, provide a cell source for drug testing, or function as a model for research into lung diseases. Induced to pluripotency through a variety of techniques, stem cells can be differentiated to alveolar epithelium through exposure to a variety of different culture conditions and growth media. The ultimate success of differentiated cells for translational medicine applications will depend on further advances in the understanding of the human lung developmental pathway, and successful application to in vitro culture. This review will focus on the major signaling pathways and molecules in lung development and the existing protocol for directed differentiation of iPSC and hESC to cells resembling respiratory epithelium in vitro.
\end{abstract}

Keywords Pluripotent stem cells $\cdot$ Differentiation $\cdot$ Lung epithelial cells

\section{Introduction}

It is estimated that about 60 million people are living with lung disease around the world, over 4 million of whom die each year $[1,2]$. These numbers are increasing, a trend which is projected to continue as the global burden of disease is increasingly dominated by noncommunicable diseases.

This article is part of the Topical Collection on Tissue Engineering and Regeneration

M. Ghaedi $\cdot$ L. E. Niklason $(\bowtie) \cdot J$. C. Williams

Departments of Anesthesia and Biomedical Engineering, Yale University, New Haven, CT 06520, USA

e-mail: laura.niklason@yale.edu
Currently, the only definitive treatment for debilitating, endstage lung diseases is orthotopic lung transplantation $[2,3]$. However, there are several problems with lung transplantation including a shortage of donor organs, the risk of graft rejection, and the taxing regimen of immunosuppressive drugs that patients must take for the rest of their lives [4, 5].

One potential future treatment for these patients is transplantation of tissue-engineered lung made from a patient's own cells. A recent approach in lung tissue engineering is to decellularize damaged or immunologically incompatible lungs to generate scaffolds that retain their topological specificity, including a perfusable vascular bed and preserved airway and alveolar geometry $[1,3,6-8]$. These scaffolds could then potentially be reseeded with epithelial and endothelial cells and transplanted into patients. One important roadblock to engineering new lung is the scarcity of human lung epithelial cells, and difficulty in the isolation and expansion of these primary cells [9-11]. Lung epithelial cells differentiated from iPSCs have the potential to be a patient-specific source of cells, potentially reducing the problem of graft rejection and eliminating the need for immunosuppressive drugs post transplantation. However, significant improvements need to be made in optimizing the cell source and scaffold before engineered lungs can be brought to the clinic, and we anticipate that it will be several decades before such engineered organs might be suitable for clinical use [10-12].

There are several types of specialized epithelial cells, including ciliated cells, club cells, basal cells, goblet cells, and alveolar epithelial type I and II cells (ATI and ATII), that line the airways and alveoli of the lung, performing a great number of vital functions. The goal in differentiating epithelial cells from iPSCs is to generate pure cell populations with the ability to expand in vitro to levels that would allow for their use in lung tissue engineering, and potentially in the development of new drugs. Moreover, these cells would be of great use in disease modeling applications [13-17]. 
The current model for lung cell differentiation is to follow the paradigm of the embryonic lung developmental pathway. Lung development in vivo is dictated by specific temporal control of signaling pathways and growth factors that have been partially, but not fully, elucidated $[10,18,19]$. Work still needs to be done to improve the consistency and yield of differentiation protocols, and to better characterize the resultant cells. With these goals in mind, this review will cover the most recent strategies and protocols for alveolar epithelial differentiation from iPSCs, to increase understanding of this quickly-moving field and its contribution to the engineering of functional lungs.

\section{What are induced Pluripotent Stem Cells (iPSCs)?}

Induced pluripotent stem cells (iPSCs) are adult cells that have been genetically reprogrammed to an embryonic stem celllike stage by the introduction of pluripotency genes and factors important for maintaining the defining properties of embryonic stem cells [20••]. In 2006, Shinya Yamanaka's lab reported for the first time that the introduction of four specific pluripotent genes could convert adult mouse fibroblast cells to pluripotent stem cells with qualities remarkably similar to embryonic stem cells. A year later, in 2007, James Thomson showed that human fibroblasts could also be genetically reprogrammed back into an embryonic-like state [21-23]. For both mouse and human models, these induced pluripotent stem cells (iPSCs) demonstrate expression of stem cell markers, formation of tumors containing cells from all three germ layers when implanted into mice, and the ability to contribute to many different tissues when injected into mouse embryos at a very early stage in embryogenesis. In vitro, they are also capable of generating many different human cell types including hepatocytes, neurons, respiratory epithelial cells, etc. Since this groundbreaking discovery, iPSC research has quickly become the foundation for a new branch of regenerative medicine [23-25, 26•].

iPSCs can be derived from skin fibroblasts or blood cells by introducing a specific set of pluripotency-associated genes, or "reprogramming factors" such as Oct4 (Pou5f1), Sox2, cMyc, and Klf4 (also dubbed "Yamanaka factors") using different methods [21]. Genes for these transcription factors were originally introduced using a lentiviral or retroviral based vector $[21,27]$. Soon after the initial discovery, researchers realized that each of the factors can be functionally replaced by related transcription factors, miRNAs [28, 29], small molecules [30], excisable vectors [31, 32], or even non-related genes such as lineage specifiers [21]. Some of these newer methods leave the cells free of transgenic material, which increases their potential for use in clinical applications. Although hiPSCs meet the criteria for pluripotent stem cells, there are differences in their gene expression profiles, compared to human embryonic stem cells (hESCs), which may affect their ability to differentiate into certain cell lines. More research needs to be done to determine whether these differences have any clinical significance, as regards both lung epithelial and other types of cellular differentiation [33].

\section{Lung Development}

The human lung is an organ with a surface area of approximately $50-100 \mathrm{~m}^{2}$, that can be used to exchange oxygen and carbon dioxide $[34,35]$. The adult lung has a complex threedimensional structure that shows major differences in the composition of the epithelium along its proximo-distal axis, transitioning from bronchial epithelial cells (ciliated and columnar cells), in proximal airways, to alveolar Type I and II cells in distal spaces. In the trachea and primary bronchi (cartilaginous airways), the luminal epithelium contains three main columnar cell types: ciliated cells, neuroendocrine cells (NE) and Clara-like cells. The latter produce secretoglobins called Clara cell secretory protein (CCSP). Basal cells (BCs), are relatively undifferentiated cells that act as stem cells in an epithelial sheet in airway. It has previously been shown that when damage occurs to the airway epithelial cells, $\mathrm{BCs}$ give rise to other specialized cells such as Clara cells and ciliated cells in the airway $[16,36]$. In the more distal airways (small bronchi and bronchioles), Clara cells predominate over ciliated cells, and there are more NE cells than in the trachea. The most distal region of the lung is organized into a complex system of alveoli that are comprised of two primary epithelial cells types: Type 1 (AT1), and Type 2 (AT2) pneumocytes. AT1 cells [37] line the majority of the alveolus and are primarily responsible for gas-exchange, while AT2 pneumocytes secrete alveolar surfactants which increase lung compliance $[13,15,38]$.

Lung arises from the endoderm, which is classically defined as the inner germ layer of the embryo. The definitive endoderm is a part of the endoderm that forms inside of the embryo (it does not contribute to extraembryonic structures), during gastrulation, and replaces the extraembryonic visceral endoderm $[19,35]$. The main endoderm derivative is the epithelial lining of the digestive tract, but it also participates in the genesis of many other visceral organs such as the liver, lungs, pancreas, and thyroid [35, 39, 40]. Definitive endoderm folds to make a gut tube and is patterned along the anteriorposterior and dorsa- ventral axis. The lung endoderm buds from the ventral side of anterior foregut endoderm to form the primitive lung bud. Soon after, the resulting lung bud starts branching and generates the respiratory tree, undergoing cell differentiation to form both the conducting airways and the terminal alveoli. Endoderm-derived cells from the lining of the lung bud differentiate into respiratory epithelium, which lines airways, and the specialized epithelium that lines the alveoli. However, the other two germ layers also contribute to the lung; the ectoderm contributes to innervation of the 
whole lung, and the mesoderm contributes to the generation of blood vessels, airway smooth muscle, pulmonary fibroblasts, cartilage, and other connective tissue in lung and trachea [39-41].

Mesenchyme has a particularly important role in epithelial specification and differentiation. During lung development, the interaction between epithelium and mesenchyme through paracrine and autocrine signals is necessary for patterning of alveoli and airway structures and proper development of lung. For branching to occur, bronchial mesoderm is required. The rate and extent of branching is proportional to the amount of mesenchyme present $[39,42]$.

The appearance of different lung cell types occurs at different times during development. In humans, cilia in ciliated cells appear in the proximal airways by 13 weeks of development in the embryo. After 16 weeks, further growth occurs by branching, elongation, and lining of airways with secretory and basal cells. By 20 weeks, characteristic lamellar bodies start to appear in AT2 cells and the AT1 cells are present [43-45]. As these cell types undergo maturation, they take on functions more closely related to their roles in adult tissue. Full maturation of cells differentiated in vitro is a challenge that continues to be addressed in the field.

\section{Early Attempts to Generate Type II Cells from Stem Cells}

Progress in generating lung epithelial cells from both ESCs and iPSC has been slower than differentiation to other lineages, such as liver and cells of the nervous system. In studies over approximately the past seven years, several laboratories have reported that both mouse and human iPSC and ESCs can be induced in culture to acquire phenotypic markers of type II alveolar epithelial cells, and more proximal airway cells, using different protocols. Because of the important physiological function of AT2 cells in vivo, and the feasibility of quantification of this function by measuring expression and secretion of surfactant molecules, several investigators have chosen to focus on the generation of AT2 cells from iPSC [13-15, 18, 46-48]. Generation of more proximal airway epithelial cells, such as basal, Clara, and ciliated cells, from ESCs or iPSCs has proven more challenging. Hence, fewer studies have targeted the differentiation of airway epithelial cells, despite the fact that diseases affecting the upper airways (such as asthma and cystic fibrosis), are more prevalent than those of distal, alveolar cells. Generation of cells with airway epithelial cell phenotypic markers has been reported following culture of the ESCs under air-liquid interface conditions [18, 19, 49, 50].

Early lung lineage differentiation protocols cultured embryonic stem cells directly in conditions that were designed for the growth of freshly isolated airway and alveolar cells from human lung. This was done with the hope that the media would promote the differentiation and survival of stem cells to mature ATII and ATI or airway cells. These protocols were able to detect some surfactant protein expression indicating the presence of ATII cells; however these cells were generally present at low levels. To overcome the low efficiency and heterogeneity in hESC differentiation, ESCs were transduced with selection markers, such as the surfactant protein C (SPC) promoter, conferring the ability to select for the derived epithelial population, yielding a purer population of cells. In a significant advance of the field, Wang and colleagues linked the SPC promoter to the neomycin resistance gene and used puromycin selection to yield a $>99 \%$ pure population of ESCderived, SPC-expressing cells [13, 48]. Following these reports, Haute and colleagues used a 20 day air-liquid interface culture to derive cells expressing CC10, SPC and SPA (surfactant protein A) [15]. Though these early attempts represented a major advance of the field, protocols guided by recapitulation of the generation of AT2-like cells from ESCs in vivo have since yielded more consistent and specific results. These stepwise differentiation protocols take cells from pluripotent stem cells, to definitive endoderm, anterior foregut endoderm, to a lung progenitor cell and then through subsequent pathways to generate cells expressing markers of either AT2 cells or other types of epithelium. Using stepwise differentiation approaches, several research groups have reported the differentiation of ESCs and iPSCs toward cells expressing markers of a range of pulmonary epithelium, including both AT2 and other airway epithelium $[18,51,52]$.

\section{In Vivo Development as a Guide for Epithelial Differentiation}

As previously noted, the embryonic lung arises from definitive endoderm; therefore, appearance of markers associated with this germ layer is an important indicator of lung, and later alveolar, lineage selection. However, it became apparent in the early days of ESC research that it is considerably harder to derive endodermal lineages than those of the mesoderm and ectodermal germ layers. Therefore, attention turned to developing means by which endodermal differentiation could be specifically enhanced, and the other two germ layers suppressed, during the very early stages of ESC differentiation, before the emergence of mature somatic lineages [35, 53, 54].

\section{Definitive Endoderm Induction in hESC and hiPSC}

A comprehensive analysis of genes responsible for endoderm differentiation has shown that mesodermal and endodermal fates are determined by different expression levels of the nodal signaling pathway [55]. Studies in mice suggest that endoderm is specified by signaling from the TGF- $\beta$ family, notably the secretory protein nodal. Studies in zebra fish also showed that nodal signaling specifies mesoderm and endoderm in a concentration- and dosage-dependent manner [56]. Among the signals that activate the nodal pathway is Wnt signaling. 
Studies in mouse embryos lacking either the nodal or $\beta$ catenin (a signal transducer in the Wnt signaling pathway) gene, failed to show primitive streak formation in the embryo. This suggests that both the nodal and canonical Wnt signaling pathways work synergistically to specify the endoderm layer $[35,57,58]$.

Many labs use knowledge of these pathways to specify definitive endoderm from stem cells in vitro. However, due to the limited availability and the high price of the nodal protein, most labs currently use Activin A instead. Activin A is a signaling protein that activates the same downstream signaling cascade as nodal to induce a definitive endoderm phenotype in embryonic stem cells. Initial experiments by Kubo [53] and Yasunaga [59] on mouse ES cells, and D'Amour [54] on human ES cells, showed that definitive endoderm can be induced efficiently from ES cells by high concentrations of Activin A in low serum conditions. The low serum environment most likely limits phosphatidylinositol 3 kinase (PI3K) activity, a condition needed for definitive endoderm formation from ES cells [60]. Using this model, different laboratories have generated protocols for endoderm induction, although the efficiencies of the protocols vary, apparently largely depending on the cell lines used [53, 54]. Analysis of several transcription factors acting downstream of nodal/Wnt signaling can also be used to measure the success of definitive endoderm-generating protocols. Forkhead transcription factors of the FoxA family and GATA factors are key transcription factors of the endodermal network. Several family members of these transcription factors are expressed in endoderm or mesoderm in most species. GATA factors are expressed in mesendoderm and are required for endoderm differentiation. Specific to vertebrates, other key components of the network downstream of Nodal include SOX17, MIX, and several related genes (such as CXCR4, c-kit, GATA4, GATA6, and EpCAM), which have been described in endoderm, and which may be used as markers for endoderm characterization [10, $18,52]$.

Anterior Foregut Endoderm Induction in hESC and hiPSC

After formation of definitive endoderm in the embryo, the endoderm folds into a primitive gut tube, from which numerous organs subsequently bud after anterior-posterior patterning. Studies using an assay of in vitro germ layer explants to characterize the signals involved in developmental specification, show that differentiation of the endoderm to different organs is directed by signals from adjacent mesoderm and ectoderm. The first step in lung formation is the appearance of primary lung buds from the ventral-lateral aspect of the foregut. These lung buds contain NKX2.1 (homeodomaincontaining transcription factor), the earliest known marker associated with lung lineage commitment [61]. However, NKX2.1 is also associated with commitment to the thyroid lineage and does not appear as a marker in the lung until the lung progenitor stage of differentiation [10,62].

Although the definitive endoderm cells derived from hESC or iPSC using high concentrations of Activin A have been presumed to be broadly multipotent, studies have shown that most anterior foregut endodermal lineages, such as thymus, thyroid, and lung epithelia, have been difficult to derive from these progenitors [10]. Green's report on human ES and iPS [10], and that of Longmire on mouse ES cells [18], have clearly demonstrated that dual inhibition of TGF- $\beta$ /BMP signaling of iPSC-derived definitive endoderm with high levels of Noggin (an inhibitor of BMP signaling), and SB141524 (a TGF- $\beta$ signaling inhibitor), led to the quantitative generation of anterior foregut endoderm from definitive endoderm. This AFE could be subsequently differentiated into cells expressing markers of thymus, lung, and thyroid [18, 19, 52].

\section{Generation of the Lung Progenitor Cells from Anterior} Foregut Endoderm

There are several molecules and signaling pathways involved in the differentiation of AFE to lung progenitor cells during development. Signaling molecules and pathways including Nodal, TGF- $\beta$ (transforming Growth Factor), BMP (Bone Morphogenetic Protein), FGF (Fibroblast Growth Factor), retinoic acid (RA), Notch, and Wnt play central roles in differentiation of the foregut endoderm into the lung [63-66]. These signaling molecules are mostly expressed in gradients leading to dose-dependent responses along the anteriorposterior axis of foregut. A better understanding of growth factors, signaling pathways, and the precise regulation of these pathways, could yield more efficient derivation of lung epithelial cells from iPSCs and hESCs in the future. The role of these currently known signaling pathways/molecules is described below.

\section{BMP}

BMPs are a family of molecules, and their signaling is active in the ventral region of the foregut endoderm in vivo and leads to an increase in NKX2.1 during anteriorization by suppressing the SOX2. During lung development, BMP4 is expressed distally, while BMP7 is expressed closer to the airway. BMP7 promotes formation of the trachea, respiratory structures, and proximal epithelium [19, 65, 67].

Wnt

Numerous studies have shown that Wnt signaling from the mesenchyme plays an important role in patterning of anterior endoderm, and is necessary to induce NKX2.1 expression in these cells in vivo [63, 68]. The canonical Wnt pathway promotes endoderm specification in mammals by stimulation of 


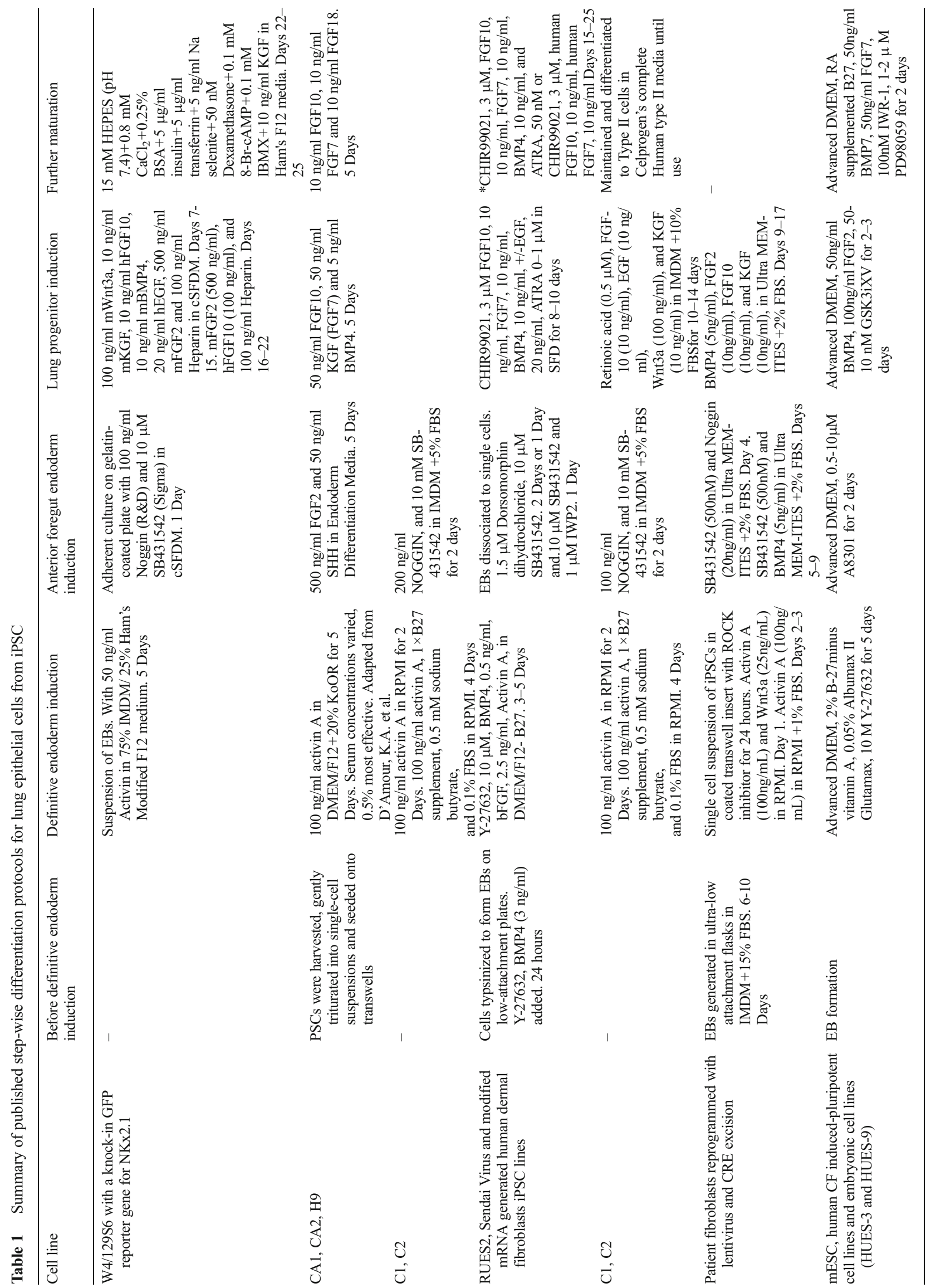




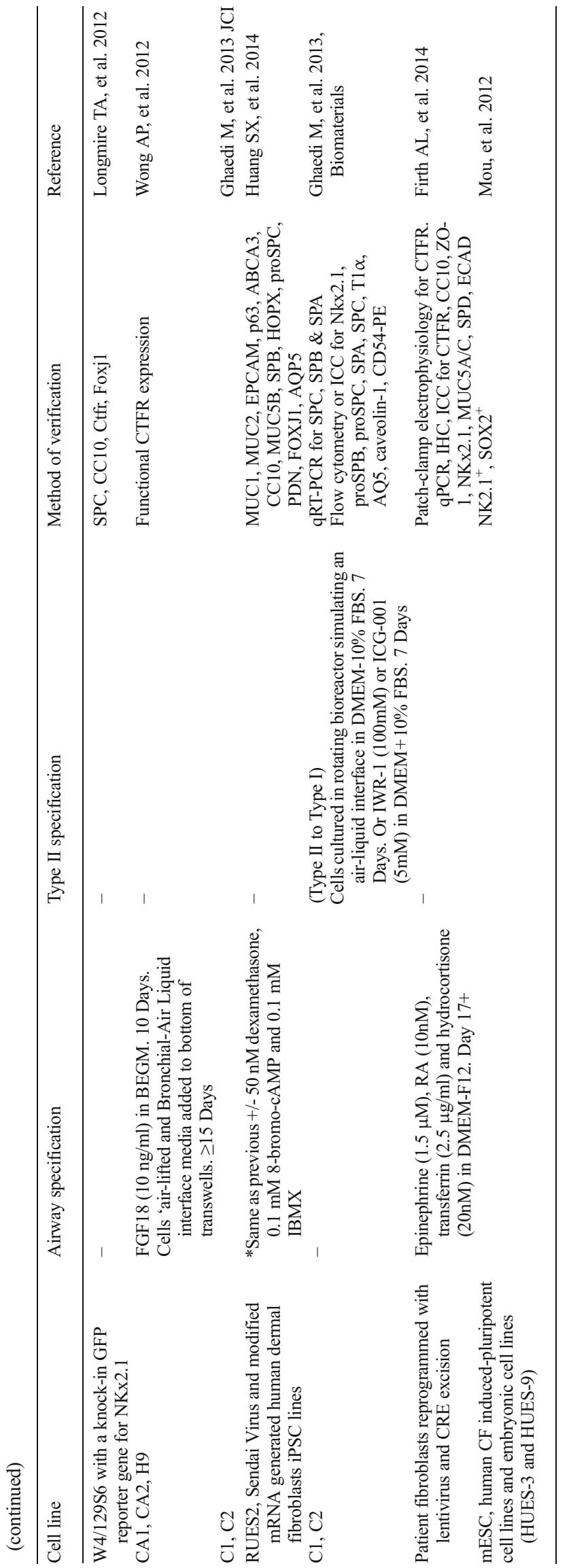

the Nodal pathway during embryogenesis. Both BMP and Wnt cooperate to regulate endoderm formation and lung specification. There is a sequential positive feedback loop between BMP and Wnt signaling, and each can activate the other. Both BMP and Wnt signaling are also activated by Nodal $[35,57]$.

\section{FGF}

Different members of the FGF family (such as FGF-10, FGF2 , and FGF-7), influence the patterning of lung along the proximal-distal axis from ventral foregut endoderm, from their locations in the mesenchyme [66]. FGF signaling, along with BMP4 and Wnt signaling, is required to specify lung endoderm from the anterior foregut. FGF signaling is mostly located at the distal branching tips. FGF-10 signaling from mesoderm is required for growth of the lung bud, while FGF-7 is more important in proximal airway formation $[19,64]$.

\section{Retinoic Acid (RA)}

This metabolite is necessary for proper lung morphogenesis and differentiation of the lung epithelium. RA signaling has a critical role in early lung bud formation and subsequent branching $[69,70]$.

During lung development, the concentration and timing of these growth factors will change at different stages. Distal FGF-2 and FGF-10 are replaced by proximal KGF (keratinocyte growth factor, which is FGF-7), during proximal-distal specification; while Wnt signaling is inhibited in the proximal stalk progenitors, but not in the distal tip [57, 68]. Retinoic acid is an essential factor for lung bud development, but RA concentration is relatively higher in the proximal stalk region, than at the distal tip region [64]. High RA signaling prevents distal lung development and favors proximal airway development [67].

By following these known signaling pathways involved in in vivo lung development, researchers are attempting to apply the same signaling pathways for the differentiation of PSCs into lung epithelium, with some degree of success $[10,18,19$, 51, 52]. In 2012, Green described a method for patterning anterior foregut endoderm-like cells derived from human ES and iPSC to alveolar type II-like epithelial cells, using a combination of BMP4, Wnt3a, FGF-10, EGF, and RA in hESC [10]. In another report, Longmire optimized the method to generate the lung progenitor cells expressing NKX2.1 by activating the combinatorial FGF, BMP, and Wnt signaling pathways in mouse iPSC [18]. Using the growth factors cocktail containing Wnt, FGF10, EGF, KGF, and RA, we were able to generate a relatively homogeneous population of AT2 like cells from human iPSC. Additionally, exposing AT-2 like cells to IWR-1, a small molecule inhibitor of Wnt/b-catenin/Crebbinding protein (CBP) transcription, changed the iPSC- 
derived AT2 like phenotype to a predominantly AT1like phenotype [71, 72].

Although most of the efforts of researchers are focused on differentiation of iPSCs and hESCs to distal epithelium, there are a few established protocols for differentiation of iPSC and hESC cells to airway epithelial cells. It has been shown by Mou H, that BMP4, FGF-2, and Wnt are necessary for induction of their NKX2.1 $1^{+}$lung progenitors that are able to mature into $\mathrm{NKX} 2.1^{+} \mathrm{SOX} 2^{+}$proximal progenitor cells and $\mathrm{NKX} 2.1^{+} \mathrm{P} 63^{+}$basal-like cells in vitro; in the presence of a growth factor cocktail containing KGF, BMP7 and a high concentration of RA [19]. In another study, Wong in 2013 showed that human pluripotent stem cells can be directed to differentiate into CFTR-functional conducting airway epithelium, by inducing the combination of Wnt, FGF, and BMP signaling pathways [25] (Table 1).

Although several groups have achieved some expression of many differentiated lung epithelial markers, including SPC, SPB (Surfactant protein B), CCSP, CFTR (cystic fibrosis transmembrane conductance regulator), $\mathrm{P} 63$, and $\mathrm{T} 1-\alpha$ (Podoplanin) [10, 19, 25, 51, 52, 73], the major remaining challenge is to derive mature and functional lung epithelial cells that are highly similar to their in vivo counterparts, and at a yield that is reasonable for screening or regeneration purposes.

\section{Conclusions}

Current protocols attempt to recapitulate the same developmental pathways that occur in vitro to differentiate the stem cells to human respiratory cells. BMP, Wnt, FGF, and nodal signaling pathways are among the most important pathways targeted for precise temporal control. However, a great deal of optimization of existing protocols has yet to be done to produce highly pure cells in large enough numbers to be of use in clinical applications. One major challenge to the field is overcoming the variable results produced by the use of different starting iPS cell lines, and trying to generate more mature lung epithelial cells from iPS cells.

Acknowledgments This work was supported by the National Institute of Biomedical Imaging and Bioengineering (NIBIB) and National Heart Lung and Blood Institute (NHLBI) (NIH 5U01HL110967-03 to MG). Dr. Niklason reports grants from United Therapeutics and NIH.

\section{Compliance with Ethics Guidelines}

Conflict of Interest Mahboobe Ghaedi and Jordana C. Williams declare that they have no conflict of interest.

Laura E. Niklason has a financial interest in Humacyte, Inc, a regenerative medicine company. Humacyte did not fund these studies, and Humacyte did not affect the design, interpretation, or reporting of any of the experiments herein. In addition, Dr. Niklason has a patent for Epithelium from iPS cells licensed to United Therapeutics.

Human and Animal Rights and Informed Consent This article does not contain any studies with human or animal subjects performed by any of the authors.

\section{References}

Papers of particular interest, published recently, have been highlighted as

- Of importance

•- Of major importance

1. Petersen $\mathrm{TH}$ et al. Bioreactor for the long-term culture of lung tissue. Cell Transplant. 2011;20(7):1117-26.

2. Petersen $\mathrm{TH}$ et al. Tissue-engineered lungs for in vivo implantation. Science. 2010;329(5991):538-41.

3. Ott $\mathrm{HC}$ et al. Regeneration and orthotopic transplantation of a bioartificial lung. Nat Med. 2010;16(8):927-33.

4. Ahmad S, Shlobin OA, Nathan SD. Pulmonary complications of lung transplantation. Chest. 2011;139(2):402-11. doi:10.1378/ chest. 10-1048.

5. McCurry KR et al. Lung transplantation in the United States, 19982007. Am J Transplant. 2009;9(Part 2):942-58.

6. Ikonomou $\mathrm{L}$ et al. Programmatic change: lung disease research in the era of induced pluripotency. Am J Physiol Lung Cell Mol Physiol. 2011;301(6):L830-5. doi:10.1152/ajplung.00255.2011.

7. O'Neill JD et al. Decellularization of human and porcine lung tissues for pulmonary tissue engineering. Ann Thorac Surg. 2013;96(3):1046-55.

8. Badylak SF et al. Engineered whole organs and complex tissues. Lancet. 2012;379(9819):943-52.

9. Wagner WR, Griffith BP. Reconstructing the lung. Science. 2010;329(5991):520-2.

10. Green MD et al. Generation of anterior foregut endoderm from human embryonic and induced pluripotent stem cells. Nat Biotechnol. 2011;29(3):267-72.

11. Ghaedi M, et al. Efficient derivation of purified distal lung alveolar epithelium from human induced pluripotent stem cells JCI, 2013: p. in press.

12. Kotton DN. Next-generation regeneration: the hope and hype of lung stem cell research. Am J Respir Crit Care Med. 2012;185(12):1255-60.

13. Ali NN et al. Derivation of type II alveolar epithelial cells from murine embryonic stem cells. Tissue Eng. 2002;8(4):541-50.

14. Rippon HJ et al. Derivation of distal lung epithelial progenitors from murine embryonic stem cells using a novel three-step differentiation protocol. Stem Cells. 2006;24(5):1389-98.

15. Van Haute L et al. Generation of lung epithelial-like tissue from human embryonic stem cells. Respir Res. 2009;10:105.

16. Rock JR, Randell SH, Hogan BL. Airway basal stem cells: a perspective on their roles in epithelial homeostasis and remodeling. Dis Model Mech. 2010;3(9-10):545-56.

17. Barkauskas CE et al. Type 2 alveolar cells are stem cells in adult lung. J Clin Invest. 2013;123(7):3025-36. doi:10.1172/JCI68782.

18. Longmire TA et al. Efficient derivation of purified lung and thyroid progenitors from embryonic stem cells. Cell Stem Cell. 2012;10(4): 398-411.

19. Mou $\mathrm{H}$ et al. Generation of multipotent lung and airway progenitors from mouse ESCs and patient-specific cystic fibrosis iPSCs. Cell Stem Cell. 2012;10(4):385-97. 
$20 . \bullet$ Inoue $\mathrm{H}$ et al. iPS cells: a game changer for future medicine. EMBO J. 2014;33(50):409-17. Discusses the use of iPSCs in disease modeling, cell transplantation, and clinical trials. These cells could lead to important changes in the future of medicine.

21. Takahashi $\mathrm{K}$ et al. Induction of pluripotent stem cells from adult human fibroblasts by defined factors. Cell. 2007;131(5):861-72.

22. $\mathrm{Yu} \mathrm{J}$ et al. Induced pluripotent stem cell lines derived from human somatic cells. Science. 2007;318(5858):1917-20.

23. Yamanaka S. A fresh look at iPS cells. Cell. 2009;137(1):13-7.

24. Inoue H, Yamanaka S. The use of induced pluripotent stem cells in drug development. Clin Pharmacol Ther. 2011;89(5):655-61.

25. Wong AP et al. Directed differentiation of human pluripotent stem cells into mature airway epithelia expressing functional CFTR protein. Nat Biotechnol. 2012;30(9):876-82.

26. Okano $\mathrm{H}$ et al. Steps toward safe cell therapy using induced pluripotent stem cells. Circ Res. 2013;112(3):523-33. Reviews recent achievements and future tasks for safe iPSC-based cell therapy, using regenerative medicine for repair strategies in the CNS as a model. Also discusses insights on safety and preclinical use of iPSCs in a cardiovascular repair model.

27. Takahashi K, Yamanaka S. Induction of pluripotent stem cells from mouse embryonic and adult fibroblast cultures by defined factors. Cell. 2006;126(4):663-76.

28. Li Z, Rana TM. Using microRNAs to enhance the generation of induced pluripotent stem cells. Curr Protoc Stem Cell Biol, 2012. Chapter 4:Unit 4A.4. doi: 10.1002/9780470151808.sc04a04s20

29. $\mathrm{Li} \mathrm{Z}$ et al. Small RNA-mediated regulation of iPS cell generation. EMBO J. 2011;30(5):823-34.

30. Kim $\mathrm{D}$ et al. Generation of human induced pluripotent stem cells by direct delivery of reprogramming proteins. Cell Stem Cell. 2009;4(6):472-6.

31. Stadtfeld $\mathrm{M}$ et al. Induced pluripotent stem cells generated without viral integration. Science. 2008;322(5903):945-9.

32. Kaji $\mathrm{K}$ et al. Virus-free induction of pluripotency and subsequent excision of reprogramming factors. Nature. 2009;458(7239):771-5.

33. Mallon BS et al. Comparison of the molecular profiles of human embryonic and induced pluripotent stem cells of isogenic origin. Stem Cell Res. 2014;12(2):376-86.

34. Tomco R. Lungs and mechanics of breathing. AnatomyOne.Amirsys, Inc. Retrieved -09-28, 2012.

35. Grapin-Botton A. Endoderm specification. SourceStemBook [Internet]. Harvard Stem Cell Institute: Cambridge (MA); 2008.

36. Rackley CR, Stripp BR. Building and maintaining the epithelium of the lung. J Clin Invest. 2012;122(8):2724-30.

37. Cheek JM, Evans MJ, Crandall ED. Type I cell-like morphology in tight alveolar epithelial monolayers. Exp Cell Res. 1989;184(2): 375-87.

38. Rawlins EL, Hogan BL. Epithelial stem cells of the lung: privileged few or opportunities for many? Development. 2006;133(13):245565.

39. Peng $\mathrm{T}$ et al. Coordination of heart and lung co-development by a multipotent cardiopulmonary progenitor. Nature. 2013;500(7464): 589-92.

40. Lewis SL, Tam PP. Definitive endoderm of the mouse embryo: formation, cell fates, and morphogenetic function. Dev Dyn. 2006;235(9):2315-29.

41. Serls $\mathrm{AE}$ et al. Different thresholds of fibroblast growth factors pattern the ventral foregut into liver and lung. Development. 2005;132(1):35-47.

42. Blanc P, et al. A role for mesenchyme dynamics in mouse lung branching morphogenesis. PLoS One, 2012: p. 7(7): e41643.

43. Rawlins EL et al. Lung development and repair: contribution of the ciliated lineage. Proc Natl Acad Sci U S A. 2007;104(2):410-7.

44. Desai TJ, Brownfield DG, Krasnow MA. Alveolar progenitor and stem cells in lung development, renewal and cancer. Nature. 2014;507(7491):190-4.
45. Chang DR et al. Lung epithelial branching program antagonizes alveolar differentiation. Proc Natl Acad Sci U S A. 2013;110(45): 18042-51.

46. Banerjee ER et al. Human embryonic stem cells differentiated to lung lineage-specific cells ameliorate pulmonary fibrosis in a xenograft transplant mouse model. PLoS ONE. 2012;7(3):e33165.

47. Samadikuchaksaraei A et al. Derivation of distal airway epithelium from human embryonic stem cells. Tissue Eng. 2006;12(4):867-75.

48. Wang D et al. A pure population of lung alveolar epithelial type II cells derived from human embryonic stem cells. Proc Natl Acad Sci U S A. 2007;104(11):4449-54.

49. Wong AP et al. Directed differentiation of human pluripotent stem cells into mature airway epithelia expressing functional CFTRTR protein. Nat Biotechnol. 2012;30(9):876-82.

50. Firth AL et al. Generation of multiciliated cells in functional airway epithelia from human induced pluripotent stem cells. Proc Natl Acad Sci U S A. 2014;111(17):E1723-30.

51. Huang SX et al. Efficient generation of lung and airway epithelial cells from human pluripotent stem cells. Nat Biotechnol. 2014;32(1):84-91.

52. Ghaedi M, et al. Efficient derivation of purified distal lung alveolar epithelium from human induced pluripotent stem cells JCI, 2012.

53. Kubo A et al. Development of definitive endoderm from embryonic stem cells in culture. Development. 2004;131(7):1651-62.

54. D'Amour KA et al. Efficient differentiation of human embryonic stem cells to definitive endoderm. Nat Biotechnol. 2005;23(12): 1534-41.

55. Thisse B, Wright CV, Thisse C. Activin- and Nodal-related factors control antero-posterior patterning of the zebrafish embryo. Nature. 2000;403(6768):425-8.

56. Hagos EG, Dougan ST. Time-dependent patterning of the mesoderm and endoderm by Nodal signals in zebrafish. BMC Dev Biol. 2007;7:22

57. Huelsken $\mathrm{J}$ et al. Requirement for beta-catenin in anterior-posterior axis formation in mice. J Cell Biol. 2000;148(3):567-78.

58. Ben-Haim $\mathrm{N}$ et al. The nodal precursor acting via activin receptors induces mesoderm by maintaining a source of its convertases and BMP4. Dev Cell. 2006;11(3):313-23.

59. Yasunaga $\mathrm{M}$ et al. Induction and monitoring of definitive and visceral endoderm differentiation of mouse ES cells. Nat Biotechnol. 2005;23(12):1542-50.

60. McLean $\mathrm{AB}$ et al. Activin a efficiently specifies definitive endoderm from human embryonic stem cells only when phosphatidylinositol 3-kinase signaling is suppressed. Stem Cells. 2007;25(1):29-38.

61. Cardoso WV, Lu J. Regulation of early lung morphogenesis: questions, facts and controversies. Development. 2006;133(9):1611-24.

62. Constam DB, Robertson EJ. SPC4/PACE4 regulates a TGFbeta signaling network during axis formation. Genes Dev. 2000;14(9): 1146-55.

63. Goss $\mathrm{AM}$ et al. Wnt $2 / 2 \mathrm{~b}$ and beta-catenin signaling are necessary and sufficient to specify lung progenitors in the foregut. Dev Cell. 2009;17(2):290-8.

64. Ameri J et al. FGF2 specifies hESC-derived definitive endoderm into foregut/midgut cell lineages in a concentration-dependent manner. Stem Cells. 2010;28(1):45-56.

65. Ninomiya $\mathrm{N}$ et al. BMP signaling regulates the differentiation of mouse embryonic stem cells into lung epithelial cell lineages. In Vitro Cell Dev Biol Anim. 2013;49(3):230-7.

66. Kaza AK et al. Keratinocyte growth factor enhances postpneumonectomy lung growth by alveolar proliferation. Circulation. 2002;106(12 Suppl 1):I120-4.

67. Morrisey EE, Hogan BL. Preparing for the first breath: genetic and cellular mechanisms in lung development. Dev Cell. 2010;18(1):823. 
68. Shu W et al. Wnt/beta-catenin signaling acts upstream of N-myc, BMP4, and FGF signaling to regulate proximal-distal patterning in the lung. Dev Biol. 2005;283:226-39.

69. Malpel S, Mendelsohn C, Cardoso WV. Regulation of retinoic acid signaling during lung morphogenesis. Development. 2000;127(14): 3057-67.

70. Desai $\mathrm{TJ}$ et al. Distinct roles for retinoic acid receptors alpha and beta in early lung morphogenesis. Dev Biol. 2006;291(1):12-24.
71. Ghaedi $\mathrm{M}$ et al. Human iPS cell-derived alveolar epithelium repopulates lung extracellular matrix. JCIJ Clin Invest. 2013;123(11):4950-62. doi:10.1172/JCI68793.

72. Ghaedi $\mathrm{M}$ et al. Alveolar epithelium differentiation of human iPS cells in a rotating bioreactor. Biomaterials. 2013. doi:10.1016/j.biomaterials.

73. Firth AL et al. Generation of multiciliated cells in functional airway epithelia from human induced pluripotent stem cells. Proc Natl Acad Sci U S A. 2014;111(17):E1723-30. 